\title{
Clinical Study on Dahuang Lingxian Decoction against Postoperative Recurrence of Cholelithiasis
}

\author{
Qianli Tang, Zhongzheng Guan \& Shanhui Gao \\ Guangxi University of Traditional Chinese Medicine \\ Nanning, Guangxi 530001, China \\ Tel: 86-771-313-7535 E-mail: htmgx@163.com
}

Xingzhong Wei \& Hai Huang

Rui Kang Hospital Affiliated to Guangxi University of Traditional Chinese Medicine

Nanning, Guangxi 530011, China

Jianrong Yang

People's Hospital of Guangxi Zhuang Autonomous Region

Nanning, Guangxi 530021, China

Yuan Yu \& Mingwei Huang

The First Affiliated Hospital of Guangxi University of Traditional Chinese Medicine

Nanning, Guangxi 530023, China

Fund Project: Project supported by National Natural Science Foundation of China in 2006(No: 30660232), Project supported by Natural Science Foundation of Guangxi Province in 2006(GKZ No:0640144)

\begin{abstract}
Objective: To observe the clinical effect of Dahuang Lingxian Decoction in preventing postoperative recurrence of cholelithiasis. Methods: 105 patients of cholelithiasis were randomly divided into 3 groups, receiving clinical treatment and 12 months observation. Respectively, 36 cases in the group of Dahuang Lingxian Decoction were treated with modified Dahuang Lingxian Decoction. 33 cases in blank group did not take any medicine of relieving gallbladder and discharging stone except the routine therapy in preoperational period; 36 cases in Xiaoyan Lidan group administered tablets of Xiaoyan Lidan. Results: After 3 treatment courses, it showed a total effective rate of $97.22 \%$ in Dahuang Lingxian Decoction group, $81.8 \%$ in blank group, and $83.3 \%$ in Xiaoyan Lidan group. Analyzed by statistics, the curative effects between 3 groups had obvious differences $(P<0.05)$, and Dahuang Lingxian Decoction group was significantly superior to blank group and Xiaoyan Lidan group $(P<0.05)$. Conclusions: Dahuang Lingxian Decoction had preferable efficacy on Cholelithiasis and it is worthy of further promotion in clinical application.
\end{abstract}

Keywords: Dahuang lingxian decoction, Postoperative recurrence of cholelithiasis, Clinical research

Cholelithiasis is one of the common and frequently-occurring surgical diseases of bile duct (Huang, Zhiqiang, 1998). Treatment of cholelithiasis is very thorny and postoperative recurrence is baffling question in surgical treatment. Reducing recurrence has been always an important project of clinical research.

Having the functions of smoothing liver and relieving gallbladder and discharging stone, the decoction is an effective prescription developed from experiences. Through previous clinical researches and animal experiment, it indicated that it could significantly reduce postoperative recurrence and residual stone rate of cholelithiasis and prevent from suffering pigment stone. In order to study it further, we selected cholelithiasis patients as the research objects and administrate red them modified decoction after surgery. The decoction was properly adjusted according to patients' TCM differentiation. 
Then we observed the postoperative recurrent rate. The study is summarized as follow.

\section{Clinical Data}

\subsection{General Information}

From December 2006 to November 2007, the research group formed from hepatobiliary departments of 3 hospitals of third grade and A level selected 105 cholelithiasis patients that all met the diagnostic criteria. They were randomly divided into 3 groups. Danghuang Lingxian decoction group (taken modified decoction according to TCM differentiation) had 36 cases, including 15 males and 21 females of between 65 and 31 years old. The average age was 44.2. The blank control group (accepted routine therapy in preoperational period but not to take any medicine of relieving gallbladder and discharge gallstone) had 33 cases, including 18 males and 15 females of between 67 and 31 years old. The average age was 43.2. The Xiaoyan Lidan group (taken tablet of Xiaoyan Lidan) had 36 cases, including 16 males and 20 females of between 65 and 32 years old. The average age was 43.8 . Analyzed by statistics, the data had no obvious difference between 3 groups $(\mathrm{P}>0.05)$

In the 105 cases, there were 27 patients of choledocholithiasis, 26 cases of hepatolithiasis, 31 patients of calculus of intra-extra hepatic duct and 21 patients of multiple calculi in different parts. They were all diagnosed on the history, symptoms, physical signs, biochemical tests, type-B ultrasound or CT examination, and finally proved through operation.

\subsection{Criteria for Patients Selection}

Inclusive Criteria: cholelithiasis patients who already had operation of cholecystectomy, choledocholithotomy, or exploratory of bile duct, who were set the T-tube of drainage so that we could observe, and who were between 31 and 67 years old without severe complications and agreed to accept the decoction and clinical observation.

Exclusive Criteria: a. unqualified cases to the inclusive criteria; b. difficulty in evaluating safety and excluding influential factors due to inadequacy of clinical information; c. incompletion of treatment course because of unsatisfactory effect and side effects; $d$. application or supplement of the other related medication or therapy because of society factors.

\subsection{Project Design}

Inter-comparison of groups and principal of complete random were adopted in research. Patients were randomly divided into Dahuang Lingxian group (testing group), blank control group and Xiaoyan Lidan group. The testing group took herbs of Dahuang Lingxian Decoction. Ingredients of the decoction: fresh Dahuang 12g, Weilingxian 30g, Mangxiao $15 \mathrm{~g}$, Jinqiancao 30g, Zhike 12g, Jineijin 6g(pulverizing and infusing separately), Zelan 10g, Caihu 12g, Yujin 12g, Cishi 15g, Huangqi 12g, Gancao 5g. Usage: boiled in water, twice per day, and 200ml each time. Add Chuanlianzi $12 \mathrm{~g}$ and Chuanxiong $10 \mathrm{~g}$ for syndrome of liver qi stagnation, Yinchen $15 \mathrm{~g}$ and Zhizi $10 \mathrm{~g}$ for syndrome of damp heat, and Goujizi15g, Beishashen 15g and Shihu15g for syndrome of deficiency of liver yin. Prune Mangxiao and Cahihu, and reduce the dosage of Dahuang to $6 \mathrm{~g}$ or change to dried preparation so as not to eliminate too much. 3 months treatment was a course and 3 courses regarded as a completed treatment. Patients in control group took tablet of Xiaoyan Lidan(produced by Guangzhou Baiyunshan Enterprise Group Co. Ltd Grant Number: ZZ-4007approval of Cantonese Health and Pharmacy(1996) No.132068). Usage: 3 times per day, 6 tablets per time, and 3 months treatment was a course and 3 courses regarded as a completed treatment.

\subsection{Statistical Approach}

PEMS3.1 statistics software was adopted to analyze serial samples, to adopt $\mathrm{T}$ test to compare between groups, and to use the type- $\chi^{2}$ to compare the groups' rate. Result of $P<0.05$ means having statistical senses.

\section{Criterion of therapeutic Effect and Result}

The standard was made by referring to the Chinese Medicine Standard for Diagnose and Efficacy of Clinical Disease and Syndrome. (Wang, Jingjing, 1993) Curative: clinical symptoms and signs were eliminated and no residual stone was found through ultrasound and CT examination; Marked Effective: clinical symptoms and signs were relieved mostly and a small amount of residual stone was found through ultrasound and CT examination; Effective: clinical symptoms and signs were partly eased, but a big amount of residual stone was found through ultrasound and CT examination; Ineffective: there were no improvement in clinical symptoms, signs and quantity of stone after treatment. Summary Results of Therapeutic Effect of the 3 groups (Table 1).

After 3 treatment courses, there were significant differences between the 3 groups $(\mathrm{p}<0.05)$. It showed effective rate of 97.22\% in Dahuang Lingxian group, of $81.8 \%$ in blank group, of $83.3 \%$ in Xiaoyang Lidan group. Obviously, Danhuang Lingxian group had superiority over blank group and Xiaoyan Lidan group.

No severe complications occurred in all 105cases after surgery. Drainage through $\mathrm{T}$ tube was fluent and clear. The $\mathrm{T}$ tube was removed away from 15 to 17 days, and 17 days on average. Meanwhile, cut healed well. As surgery succeeded 
in a rate of $100 \%$, there were no differences between the 3 groups $(P>0.05)$. Before removing the $\mathrm{T}$ tube, contrast examination along it was carried out after $2 \sim 3$ weeks of surgery. Ultrasound reexamination was done after 3 months. Results showed in Table. 2

The results suggested that effectiveness of Dahuang Lingxian group was superior to blank group and Xiaoyan Lidan group and recurrence rate and residual stone rate was lower than the later 2 groups after 3 months of surgery. It also indicated that the decoction might enhance the curative effect of operation of cholelithiasis and reduces the possibility of residual stone and recurrence. Not only might the decoction help patient escape from repetitious surgery, but also it might resolve to some extent this big issue of surgery.

\section{Discussions}

As cholelithiasis has a long disease process without any apparent symptoms at the early stage, sufferer would not come to hospital until clinical manifestations presented, such as abdominal pain, cholecystalgia, fever, jaundice, hyperleukocytosis, hepatic dysfunction, etc. Although there's a decline incidence of cholelithiasis in recent years, it still accounts for $16.1 \%$ of these kind conditions. ${ }^{[4]}$ Currently, surgery is the main management for it, but there is a high rate of residual stone and recurrence. (Lai, Jiaming, 2002) We conducted our research program by observing and comparing the Dahuang Lingxian decoction modified to TCM syndrome, and Xiaoyan Lidan group and blank group.

It's believed in TCM that gallbladder is attached to liver and liver regulates free movement of qi, which includes fluent excretion of bile. Symptom and cause, reducing and reinforcing, deficiency and excess must be contemporaneously considered according to the characteristics of cholelithiasis. Unblocking or purging is the general principle among various methods, for unblocking leads to painless. Specifically, Yue Meizhong, the modern famous physician, summarized 4 steps of transforming, removing, flushing, and dischatging. The writer adopted a main principal of smoothing liver and relieving gallbladder, assisting with clearing damp-heat, invigorating spleen and harmonizing stomach, resolving stasis to eliminate pain, etc., and nourishing spleen to replenish qi and yin. The decoction adopted according this theory and modified according to patients' TCM syndrome heals the condition by discharging stones and returns it to a silent period. Syndrome identification and treatment formulation make full use of the advantages of TCM. The mechanism may be that Jinqiancao Weilingxian and Dahuang, etc., act to significantly increase the bile acid and decrease bilirubin and affect the formation of the Bilirubin stones (Tang, Qianli, 2002). Those herbs, with function of resistance to cholecystitis, promoting of biliation and resolving congestion of bile, has good effects on cholelithiasis, especially on the postoperative recurrence of cholelithiasis. Huang Mingwei, etc., found that Dahuang Lingxian decoction had the effect of prevention of pigment stones, which may relate to the multi-functions of protecting the liver cell and gallbladder tissue, normalizing the metabolism of bile, and inhibiting the growth of bacteria and so on. Although bacterial infection could not be an original and inducing factor of cholelithiasis among the condition of chronic liver injury, it can increase the possibility of stone formation.

Complex prescription of Chinese herbs, with advantages of contraindicative less, safe, and effective, has fewer side effects and intends to be accepted by patient. It has showed perceived benefits to dissolve and discharge stone and achieved significant clinical effects. In recent years, Uchiyama $\mathrm{K}$ has decreased rate of residual stone to $2.4 \sim 10.0 \%$ by using choledochoscope and percutaneous transhepatic cholangioscopy. (Huang, Zhiqiang, 2000) Combination of medication and fibercholedochoscope plays an important role in enhancing the surgical effect of hepatolith. (Uchiyama $\mathrm{K}$, 2002) By combining the herbs with new technology of endoscope or choledochoscope, percutaneous transhepatic cholangioscopy and interventional treatment of western medicine, herbs could act directly on nidus and make full use of the functions of dissolving and discharging stone to enhance curative effect and reduce the recurrence of cholelithiasis. Combination of Chinese herbs and new technology has great prospects in the treatment of cholelithiasis. Up to present, Chinese scientists have found many new treatment targets of cholelithiasis. We should explore new fields in cholelithiasis for Chinese complex prescription by connecting Chinese medicine research to these new subjects (Zuo, yutong, 2005).

Postoperative residual stone in bile duct is a long-standing surgical issue for cholelithiasis. It still has great practical significance. Under the developing of high technologies, it might get resolved. Considering the unbalanced development of technology in different area in our country, it's worthwhile playing more emphasis on traditional technologies and effective experiences, and it's more valuable to research and promote them.

\section{References}

Huang, Zhiqiang, (1998). Modern Surgery of Biliary Tract M, the 1st publication: Shanghai:Shanghai Science and technology literature Publishing House, 386-398.

Tang, Qianli., Liu, Zujun and Wu, Songhe,.etc. (2002). A Clinical Observation of Dahuang Lingxian Capsule for Decreasing Residual Stone Rate and Recurrence Rate of Cholelithiasis After Operation J. New Journal of Traditional Chinese Medicine, 34 (6):17-19. 
Huang, Mingwei,. Tang, Qianli and Wu, Songhe., etc. (2005). Mechanism of Dahuang Lingxian Decoction on Preventing Formation of Pigmental Stones J. CHINESE JOURNAL OF INTEGRATED TRADITIONAL AND WESTERN MEDICINE ON DIGESTION, 13 (4): 248- 250.

Wang, Jingjing and Long, Junjie. (1993). The China Criteria for Diagnosis and Curative Effect of Clinical Disease S. Changsha: Hunan Science and Technology Publishing House, 266.

Lai, Jiaming and Liang, Lijian. (2002). Emphasis on Liver Lobectomy for Intrahepatic Calculus J. NEW JOURNAL OF TRADITIONAL CHINESE MEDICINE, 10 (1): 4-5.

Yu, Yuan., Tang, Qianli and He, Jun., etc. (2007). To Build the Cholelithiasis Model in Rabbits with CCl4 and Research the Pathogenenesis of Cholelithiasis J. CHINA MEDICAL HERALD, 4 (12): 106- 108.

Huang, Zhiqiang. (2000). The History, Actualities and Strategy to the Postoperative Residual Stone of Bile Duct J. CHINESE JOURNAL OF PRACTICAL SURGERY, 20 (9):515-516.

Uchiyama K, OnishiH, TaniM, et al. (2002). Indication and Procedure of Treatment of Hepatolithiasis J. Arch Surg, 137 (2):149-153.

Zuo, yutong., Gao, Wenyuan., Jia, wei., Duan, Hongquan and Xiao, Peigen. (2005). Prevention and Treatment for Cholelithiasis by Chinese Medicine J. China Journal of Chinese Materia Medica, 29 (9): 832 834.

Table 1. Therapeutic Effect in the 3 Groups

\begin{tabular}{lccccc}
\hline \multicolumn{1}{c}{ group } & $\mathrm{n}$ & Curative & Marked effective & Effective & Ineffective \\
\hline Dahuang Lingxian group & 36 & 20 & 10 & 5 & 1 \\
Blank control group & 33 & 14 & 10 & 3 & 6 \\
Xiaoyan Lidan group & 36 & 17 & 10 & 3 & 6
\end{tabular}

Notice: comparison between Dahuang Lingxian group and blank group, $P=0.035<0.05$; Comparison between Dahuang Lingxian group and Xiaoyan Lidan group, $P=0.047<0.05$

Table 2. Summary of Residual Stones after Surgery

\begin{tabular}{|c|c|c|c|c|c|}
\hline Group & $\mathrm{n}$ & extrahepatic & intrahepatic & Miscibility & $\begin{array}{l}\text { The number of case found residual } \\
\text { stone in } 3 \text { months after surgery }\end{array}$ \\
\hline $\begin{array}{l}\text { Dahuang Lingxian } \\
\text { group }\end{array}$ & 36 & 25 & 5 & 6 & 1 \\
\hline Blank control group & 33 & 22 & 4 & 7 & 6 \\
\hline Xiaoyan lidan group & 36 & 24 & 10 & 2 & 6 \\
\hline
\end{tabular}

Notice: comparison between Dahuang Lingxian group and blank group, $P=0.034<0.05$; Comparison between Dahuang Lingxian group and Xiaoyan Lidan group, $P=0.048<0.05$ 